\title{
A pressure-based method for monitoring leaks in a pipe distribution system: a review
}

\begin{abstract}
Leakage from a pipe network possibly poses significant environmental destruction and economic losses due to the release of potential energy. While the pipe network may be planned and constructed to satisfy the requirements of rigorous conditions, it is quite hard to avoid the subsequent appearance of leakages in a pipeline during the system's lifetime. Pressure leak detection enables a fast and reliable action response which is necessary to minimise the damage. Many leak detection approaches have been previously suggested. These methods basically depend on numerical modelling and transient analysis, such as inverse transient analysis, time domain analysis and frequency domain analysis, the negative pressure method, etc. Many methods build upon the analysis of the variation of measured pressure, such as the pressure residual vector method. Hydraulic leak detection has the important advantage of being less costly and has a faster response compared to other leak detection approaches. In this work, various leak detection methods based on pressure are listed and the analysis is reviewed. Both steady state and unsteady state conditions are discussed. The advantages and disadvantages of each approach are mentioned. In addition, methods are included that are suitable for use in both the oil and water industries.
\end{abstract}

Keyword: Pipe leak; Pipe roughness; Flow condition; Unsteady friction factor; Pipeline distribution system 